\title{
Automatic Segmentation of Abdominal Aortic Aneurism (AAA) By Using Active Contour Models
}

\author{
Rifki Kosasih ${ }^{1}$ \\ ${ }^{1}$ Computational Mathematics Study Center, Gunadarma University, Indonesia \\ Email: ${ }^{1}$ rifki_kosasih@staff.gunadarma.ac.id
}

\begin{abstract}
Abdominal aortic aneurysm (AAA) is a disease that is caused by dilation of the aortic wall. Dilation of the aortic wall will affect the size of the diameter of lumen and the aorta. In this study we use $\mathrm{T} 1$ and $\mathrm{T} 2$ images on 4 patients with AAA which generated from MR Imaging to calculate the diameter of the abdominal aortic aneurysm (AAA). To calculate the diameter of lumen and the aorta, the first step is image registration using Laplacian eigenmap method. After that we propose an automatic segmentation method on region of the aorta by using active contour models to get the contour of lumen and the aorta. The last step, we calculate the diameter of lumen and the aorta by using contour of lumen and the aorta. In our experiment, active contour model is very good method for segmentation AAA. In the result, our proposed model give the accuracy rate of lumen is $96.41 \%$ and accuracy rate of aorta is $95.22 \%$.
\end{abstract}

Keywords: Abdominal aortic aneurysm, MR Imaging, Laplacian Eigenmap, Active Contour Models, lumen

\section{INTRODUCTION}

Abdominal aortic aneurysm (AAA) consists of two parts. The inside is known as Lumen, a place where the blood flows and the outside is known as thrombus which attaches in the aortic wall. Thrombus is formed through a process called thrombosis. Thrombosis occurs when platelets attach to the surface of aortic wall. If the blood flows, then platelets will attach to the aortic wall. Consequently can be formed a mass that stands out in the aortic wall, and will cause dilation of the aorta. Dilation of the aortic wall is called an aneurysm.

If the thrombus enlarge, it will cause the dilation of aortic wall and will affect the size of the diameter of the lumen. Abdominal aorta usually have diameter about $2 \mathrm{~cm}$. But it can widen into more than $5.5 \mathrm{~cm}$ [1-2]. The dilation of the aortic wall is caused by weakening of the aortic wall because of blood pressure through aorta. If a large aneurysm occurs, it will cause rupture. In a survey in the United States, rupture occurred about $2 \%$ of patients with AAA with a diameter less than $4 \mathrm{~cm}$. Meanwhile rupture occurred more than $25 \%$ of patients with AAA with a diameter of $5 \mathrm{~cm}$ [1].

Several studies have been done to identify the AAA such as Mussa in 2015, which stated a large AAA surgery indication if the diameter $>5.5 \mathrm{~cm}$ or if the diameter of the growth rate value is greater than $1 \mathrm{~cm} /$ year [3]. AAA generally considered to be observed when diameter of aorta reaches or exceeds $3 \mathrm{~cm}$. So it is very important to know diameter of the AAA. 
The first step in this research is the detection lumen and aorta. Magnetic resonance imaging (MRI) is one of digital examination tools used in the aorta detection. One of the images which is produced by MRI is T1 and T2 weighted images. At T1 and T2 images there are differences in the position of aortic aneurysm as shown in Figure 1, so we need a method that can normalize the position of $\mathrm{T} 1$ and $\mathrm{T} 2$ weighted images such that we can do detection or automatic segmentation [4].

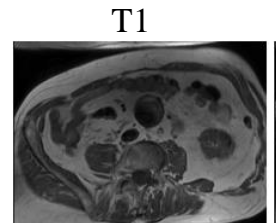

(a)

$\mathrm{T} 1$

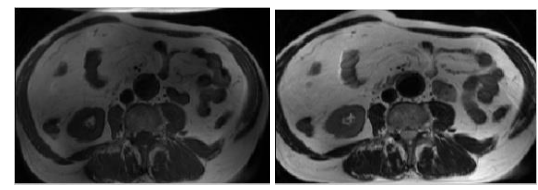

(c)
$\mathrm{T} 2$

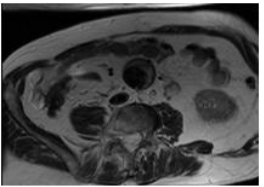

T2

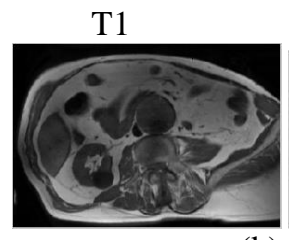

(b)
$\mathrm{T} 2$

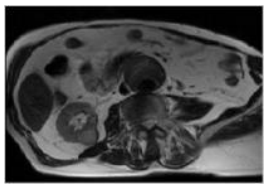

$\mathrm{T} 2$

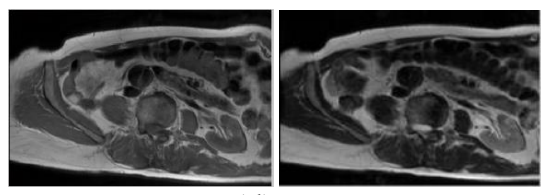

(d)

Figure 1. Original Image on 4 Patiens AAA ( (a) - (d) )

In this research we use Laplacian eigenmap method. Laplacian eigenmap is a method used to find the same structure of two or more images. Kosasih et al found that Laplacian eigenmap is a very good method to determine optimal position in AAA as shown in Figure 2 [4].

$\mathrm{T} 1$

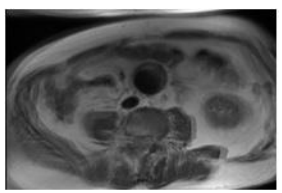

(a)

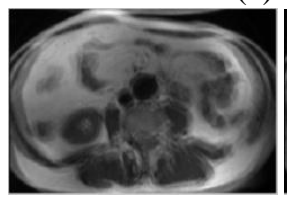

(c)
$\mathrm{T} 2$
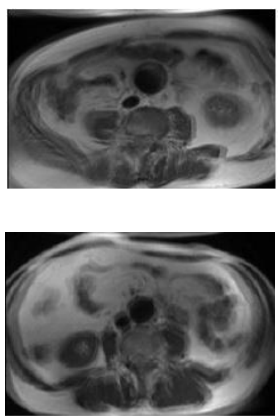

$\mathrm{T} 1$

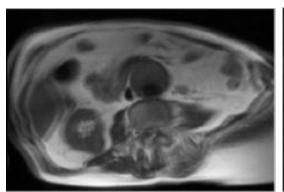

(b)

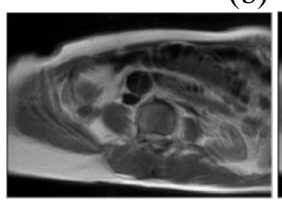

(d)
$\mathrm{T} 2$
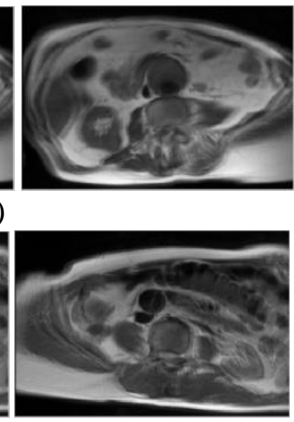

Figure 2. Laplacian Eigenmap image on 4 Patiens ((a) - (d))

In this study we performed a segmentation of the aortic and lumen area. Furthermore, we calculate maximum diameter of the aortic and lumen. In previous studies have been conducted in aortic aneurysm segmentation by Karyati. Karyati get the value of the maximum diameter of the aorta but there have been limited studies concerned on manual segmentation is shown in Figure 3 [5]. 
The weakness of manual contour is needs time longer and needs a high concentration to get contour lumen and aorta. Therefore, the objectives of this research are to perform automatic segmentation methods in the area of the aorta using active contour models.

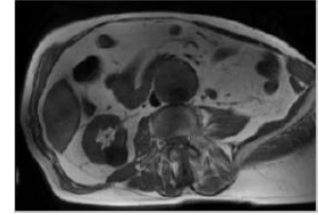

(a)

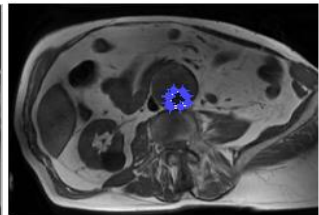

(b)

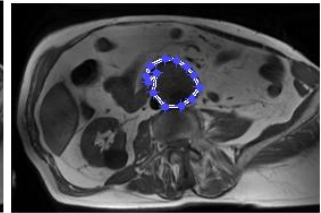

(c)

Figure 3. Manual segmentation on lumen and the aortic area a. Input Image. b. Lumen area. c. Aorta Area

\section{METHODS}

In this section, the process of segmentation to be discussed. The image of Abdominal Aortic Aneurysm was collected using MRI including T1 and T2 weighted derived from 4 patients (Figure 1). Each image was initially rescaled to compensate the different scaling of the intensity values. After that, we apply Laplacian Eigenmap to find the optimal position of aortic aneurysm. Laplacian Eigenmap is used to extract information from a set of data using the maximum eigenvector [6]. The result of Laplacian eigenmap as shown in Figure 2. After getting the optimal position of aortic aneurysm, we use an active contour model for segmentation of the lumen and aorta.

\subsection{Image Segmentation Using Active Contour Models}

Image segmentation is a fundamental problem in the field of image processing and objects recognition in computer vision [7]. Segmentation is a method to divide an image into several areas [8]. Lots of segmentation techniques that can be used, one of which is a method of active contour models (ACMs). Active contour models are one of a very good method for segmentation [9]. The basic idea of active contour models is the evolution of the curve with some constraints to extract the desired object. Based on the constraints, ACMs method is divided into two types: edge-based models and regionbased models [10-11].

Edge-based models are popular GAC models that use a gradient to construct edge stopping function to stop the evolution of the boundary contour of the object, but if the boundary of this method is weak or non-existent, then it becomes less good [12].

Another method is the method of region-based models. The method of region-based models has many advantages compared to edge-based models. method of region-based models utilize statistical information within and outside the contour to control the evolution and has a better performance expected in a weak edge or without edges [13]. One popular method is Chan Vese models based on the Mumford Shah segmentation techniques [14].

\subsection{Chan Vese Models}

Chan vese models (CV models) are special cases of the problem Munford Shah. Given image, $I \in \Omega, \mathrm{CV}$ models formulated by minimizing the following energy function: 


$$
\begin{aligned}
& E=\mu \cdot \operatorname{Length}(C)+v \cdot \operatorname{Area}(\operatorname{inside}(C))+\lambda_{1} \int_{\text {inside }(C)} \mid I(x, y)- \\
& \left.c_{1}\right|^{2} d x d y+\lambda_{2} \int_{\text {outside }(C)}\left|I(x, y)-c_{2}\right|^{2} d x d y
\end{aligned}
$$

where: $\mu \geq 0, v \geq 0, \lambda_{1}, \lambda_{2}>0$ and $c_{1}, c_{2}$ is a constant which is the average intensity inside and outside the contour, with formulations of Level set as follows:

$$
\begin{gathered}
\mathrm{C}=\{(\mathrm{x}, \mathrm{y}) \in \Omega \mid \phi(\mathrm{x}, \mathrm{y})=0\} \\
\text { inside }(\mathrm{C})=\{(\mathrm{x}, \mathrm{y}) \in \Omega \mid \phi(\mathrm{x}, \mathrm{y})>0\} \\
\text { outside }(\mathrm{C})=\{(\mathrm{x}, \mathrm{y}) \in \Omega \mid \phi(\mathrm{x}, \mathrm{y})<0\}
\end{gathered}
$$

by minimizing equation (1) is obtained that $c_{1}, c_{2}$ are:

$$
\begin{array}{r}
c_{1}(\phi)=\frac{\int \mathrm{I}(\mathrm{x}, \mathrm{y}) \mathrm{H}(\phi) \mathrm{dxdy}}{\int \mathrm{H}(\phi) \mathrm{dxdy}} \\
\mathrm{c}_{2}(\phi)=\frac{\int \mathrm{I}(\mathrm{x}, \mathrm{y})(1-\mathrm{H}(\phi)) \mathrm{dxdy}}{\int(1-\mathrm{H}(\phi)) \mathrm{dxdy}}
\end{array}
$$

Algorithm of active contour models which can be seen in the following Algorithm:

Algorithm: level set segmentation method

Input: Read data from the image Laplacian ( $\mathrm{T} 1$ and $\mathrm{T} 2$ relaxation image)

Output: T1 and T2 relaxation image with contours of the aorta and a lumen

1. Initialize the energy level set function $\phi$ with the initial value contour by clicking once on the lumen area with the mouse

2. Search foreground and background index

3. Calculate the value of $c_{1}$ and $c_{2}$

4. Calculate the value of the new Energy

5. normalize Energy

6. Check the condition:

$$
\begin{aligned}
& \text { IF }\left|\phi^{n+1}-\phi^{n}\right|<\Delta t \cdot h^{2} \text { or I }>\text { iterasi } \\
& \text { End }
\end{aligned}
$$

Else repeat back to step 4

7. Get the lumen and aortic contour.

\subsection{Morphological Operation}

Morphological operation is an operation imposed on a binary (black and white) image to change the structure of the object shape contained in the image. Core morphology operation involves two arrays of pixels. The first array is the image to be subjected to morphological operation, while the second array is named as the kernel or structuring element [15]. There are three morphological operations used in this study. The dilation operation is commonly used to obtain a widening effect on pixels of value 1 . The dilation operations is defined in equation (3).

$$
A \oplus B=\{z \mid z=a+b, \text { dengan } a \in A \text { dan } b \in B\}
$$


The erosion operation has the effect of minimizing the image structure. The erosion operation is defined in equation (4) [16].

$$
A \Theta B=\left\{p \in Z^{2} \mid(a+b) \in I, \text { untuk setiap } b \in B\right\}
$$

The opening operation is an erosion operation followed by a dilation using the same structural elements. This operation is useful for smoothing the contour of the object and removing the entire pixel in an area that is too small [17].

Definition of opening operation as follows:

$$
A \text { о } B=(A \Theta B) \oplus B
$$

\subsection{Metric Evaluation}

In this study, an evaluation of the model is done by examining the level of accuracy obtained from manual segmentation with automatic segmentation. The formula of Accuracy can be seen in equation (6) [18].

$$
\begin{aligned}
& \text { Accuracy }=\left(1-\left|\frac{\mathrm{y}_{\mathrm{i}}-\widehat{\mathrm{y}}_{\mathrm{i}}}{\mathrm{y}_{\mathrm{i}}}\right|\right) \times 100 \% \\
& \text { Average of Accuracy }=\frac{\sum_{\mathrm{i}=1}^{\mathrm{N}}\left(1-\left|\frac{y_{i}-\widehat{y}_{i}}{y_{i}}\right|\right) \times 100 \%}{\mathrm{~N}}
\end{aligned}
$$

Where $y_{i}$ is Diameter Manual, $\hat{y}_{i}$ is Diameter Automatic and $N$ is the number of dataset testing.

\section{RESULT AND DISCUSSION}

In this section, we will discuss about automatic segmentation using active contour models (Proposed Model). In this research, we will find an area and diameter of the lumen and aorta. Based on the algorithm of active contour models, result of image segmentation of AAA on patien 1 as shown in Figure 4.

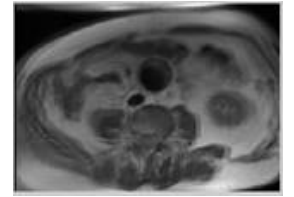

(a)

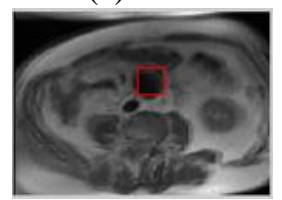

(e)

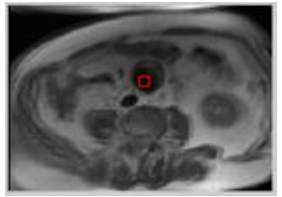

(b)

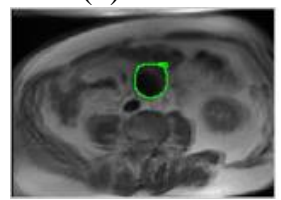

(f)

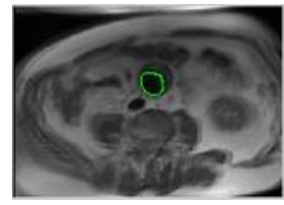

(c)

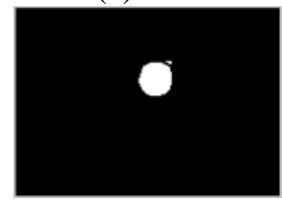

(g)

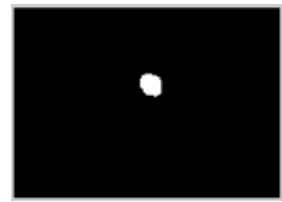

(d)

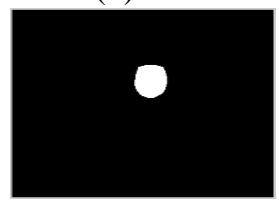

(h)

Figure 4. Automaticaly segmentation on patient 1 using proposed model 
seen in row 4 . The next step is to perform automatic segmentation to obtain the aortic region as in Table 2 .

Table 2 is the aortic segmentation process on patients 2, 3 and 4 . The process segmentation in the aorta is similar to the process of segmentation in the lumen. The first step, we make the initial box $\mathrm{A}(\mathrm{x} \pm 5, \pm 5 \mathrm{y})$ which can be seen in the first row. The results of segmentation are aortic contours which can be seen in the second row. After that, we create a mask to calculate the diameter of the aorta which can be seen in row 3. To get a better aortic region, the morphological process is used to eliminate regions outside the aorta that can be seen in row 4.

After getting the contours of lumen and the aorta, we will calculate the value of the maximum diameter of lumen and aorta. Furthermore, the calculation results were compared with the results of another researcher. Comparison of the maximum diameter of the automatic segmentation results with manual segmentation performed another researcher is shown in Table 3.

Table 2. The result of automatic segmentation on aortic area on patient 2, 3 and 4 .

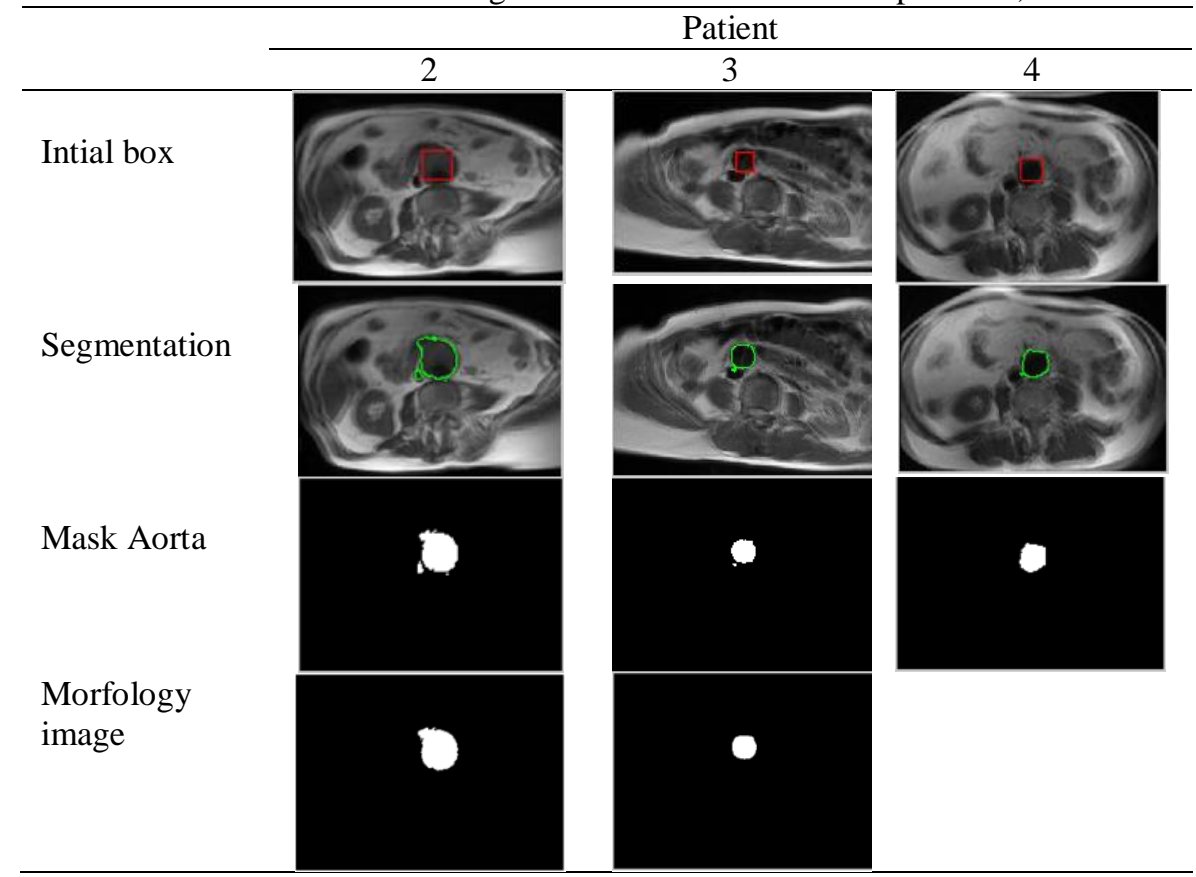


Table 3. Diameter of lumen and aorta using proposed model (automatic segmentation and manual segmentation

\begin{tabular}{ccccccc}
\hline Name & $\begin{array}{c}\text { D Lumen } \\
(\mathrm{mm}) \\
\text { Manual }\end{array}$ & $\begin{array}{c}\text { D Lumen } \\
(\mathrm{mm}) \\
\text { automatic }\end{array}$ & $\begin{array}{c}\text { Accur } \\
\text { acy } \\
(\%)\end{array}$ & $\begin{array}{c}\text { D Aorta } \\
(\mathrm{mm}) \\
\text { Manual }\end{array}$ & $\begin{array}{c}\text { D Aorta } \\
(\mathrm{mm}) \\
\text { automatic }\end{array}$ & $\begin{array}{c}\text { Accur } \\
\text { acy } \\
(\%)\end{array}$ \\
\hline P 1 & 33.34 & 33.90 & 98.32 & 46.36 & 47.93 & 96.61 \\
P 2 & 23.64 & 23.35 & 98.77 & 60.12 & 59.80 & 99.46 \\
P 3 & 23.34 & 24.71 & 94.13 & 34.78 & 35.29 & 98.53 \\
P 4 & 23.63 & 24.95 & 94.41 & 30.97 & 35.22 & 86.27 \\
Average of Accuracy from & 96.41 & $\begin{array}{c}\text { Averrage of Accuracy } \\
\text { Lumen }\end{array}$ & 95.22 \\
\multicolumn{4}{c}{ from Aorta } \\
\hline
\end{tabular}

In Table 3, the first column is the diameter of the lumen obtained from the manual segmentation and the second column is the diameter of the lumen is obtained from the automatic segmentation using the proposed model. After that the fourth column is the diameter of the aorta is obtained from the manual segmentation and the fiveth column is the diameter of the aorta is obtained from the automatic segmentation using the proposed model.

Based on Table 3 it can be seen that the average of accuracy of the measurement of the lumen diameter is $96.41 \%$ and the average of accuracy of the measurement of the aortic diameter is $95.22 \%$.

\section{CONCLUSION}

Measurement of the diameter of the lumen and the aorta in patients with AAA is one way to observe the growth of abdominal aortic aneurysm. AAA was visible when the diameter of the aorta had reached $3 \mathrm{~cm}$. In this study we used 4 patients with AAA. We propose an automatic segmentation by using active contour models to get the contour of the lumen and aorta. The contour is used to calculate the diameter of the lumen and aorta. In this research, the average of accuracy of the measurement of the lumen diameter is $96.41 \%$ and the average of accuracy of the measurement of the aortic diameter is $95.22 \%$. In the future study, we will do extraction on region of lumen to obtain information about the direction of blood flow in the lumen.

\section{REFERENCES}

[1] Beckman, J. A. (2006). Aortic aneurysms: pathophysiology, epidemiology, and prognosis in: M.A. Creager, V.J. Dzau, J. Loscalzo (Eds). Philadelphia : Elsevier Inc.

[2] Riminarsih, D., Karyati, C. M., Mutiara, A. B., Ernastuti., and Wahyudi, B. (2016). Sagittal Image Segmentation from Patients with Abdominal Aortic Aneurysms. TELKOMNIKA, 14(3), 1105-1112.

[3] Mussa, F. F. (2015). Screening for Abdominal Aortic Aneurysm. J Vasc Surg, 62(3), 774-778.

[4] Kosasih, R., Madenda, S., Karyati, C. M., and Lussiana. (2015). Determination the Optimal Position from T1 and T2 Weighted MR Imaging of the Abdominal Aortic Aneurysm. Adv. Sci. Eng. Med, 7(10), 915-919. 
[5] Karyati, C. M. (2013). Rekonstruksi 4D (3D+Waktu) Citra Aliran Darah Pada Pasien Aneurisma Aorta Abdminalis dengan Kategori Thrombus dari Hasil Pemeriksaan MRI. PhD Thesis. Gunadarma University.

[6] Belkin, M., and Niyogi, P. (2003). Laplacian eigenmaps for dimensionality reduction and data representation. Neural Computation, 15(6), 1373-1396.

[7] Zhang, J., Xie, C., Song, L., Li, R., and Chen, H. (2016). Robust Image Segmentation Using LBP Embedded Region Merging. TELKOMNIKA, 14(1), 368-377.

[8] Kusanti, J., and Santosa, Y. Z. (2016). Comparison of Patterns Shapes and Patterns Texture for Identification of Malaria Parasites in Microscopic Image. Scientific Journal of Informatics, 3(2), 109-118.

[9] Chan, T. F., \& Vese, L. A. (2001). Active Contours Without Edges. IEEE Transactions On Image Processing, 10(2), 266-277.

[10] Hou, L. (2014). Color Remote-Sensing Image Segmentation Based on Improved Region Filter. Journal of Multimedia, 9(9), 1128-1134.

[11] Kashyap, R., and Tiwari, V. (2017). Energy-Based Active Contour method for Image Segmentation. Int. J. Electronic Healthcare, 9(2), 210-225.

[12] Tjandrasa, H., Wijayanti, A., and Suciati, N. (2012). Optic Nerve Head Segmentation Using Hough Transform and Active Counturs. TELKOMNIKA, 10(3), 531-536.

[13] Zhang, K.., Zhang, L., Song, H., and Zhou, W. (2010). Active Contours with Selective Local or Global Segmentation: A New Formulation and Level Set Method. Image and Vision Computing, 28(4), 668-676.

[14] Mumford, D., and Shah, J. (1989). Approximation by piecewise smooth functions and associated variational problems. Commun. Pure Appl. Math, 42, 577-685.

[15] Goyal, M. (2011). Morphological Image Processing. International Journal of Computer Science and Technology, 2, 161-165.

[16] Shih, F. Y. (2009). Image Processing and Mathematical Morphology. New York: CRC Press.

[17] Lestari, D. P., Madenda, S., Ernastuti., and Wibowo, E. P. (2017). Comparison of Three Segmentation Methods for Breast Ultrasound Images Based on Level Set and Morphological Operations. International Journal of Electrical and Computer Engineering (IJECE), 7(1), 383-391.

[18] Kumar, B. A., Jairam, R., Arkatkar, S. S., and Vanajakshi, L. (2019). Real time bus travel time prediction using k-NN classifier. Int. J. Transp. Res, 11(7), 362372. 